\section{All the way through mammalian skin}

Sam Shuster

\section{Mammal Skin.}

By V.E. Sokolov.

University of California Press: 1983.

Pp.695. £61.50, \$72.50.

AFTER many dark years, the academic wilderness of the outer cover has been blown and biomedical scientists are coming out of the warmth of internal prying to the rewarding uncertainties of the cold surface. The spate of cutaneous publishing is part of the celebration and in that sense it may be churlish to ask for sober assessment rather than whoopee.

As might have been expected from a field in which the drive has mostly been pragmatic (disease, fur and pelt production), some topics have been dealt with rather more than others. As might also be expected of an organ whose great complexity is the unique issue of a commune rather than a marriage of other tissues, expertise can easily be maintained in one aspect side by side with the deepest oblivion of neighbourhood experts working on a different topic not a $\mu$ or two away. From this it follows that most large skin texts are multi-author jobs and they, and we, all suffer from it. The thrust of this over-long

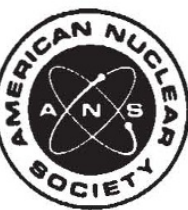

\section{AMERICAN NUCLEAR SOCIETY'S PUBLICATIONS}

\section{Complete Nuclear}

Publications and Services Worldwide.

Inquire about specific interests.

For Free Publications and Services Catalog, contact:

\section{American Nuclear Society 555 N. Kensington Avenue La Grange Park, IL 60525 U.S.A.}

welcome to this singular book must by now be apparent: we have for the first time in the English language a notably unique and personal account of the comparative structure of the mammalian skin.

There is a short general introduction on structure followed by a systematic description covering the entire immense range of mammalian skin, the book ending with a section on adaptive changes. The whole is so exhaustive that you can follow your pet themes right through their evolutionary vicissitudes, and, if you've the stamina left, crack your equally pet theories against reality: you will not travel far without finding some extraordinary and interesting new facts which, at very least, will give you a clutch of new experiments for students. This then is an old-fashioned work of scholarship the like of which is all too rarely seen.

Nonetheless there are some serious defects. The thinking, information and methodology are essentially of the 1960s and earlier, although the original 1974 Russian edition is said to have been updated (in compensation it is salutary to be reminded how well simple methods work - for instance the evidence for the thermo-regulatory function of the fins of certain aquatic mammals); some of the data is thin; and there are some surprising omissions - coat colour and pattern is an opportunity lost. Yet despite the many flaws, including a rudimentary index and indescribably bad photomicrographs, this is an outstanding contribution to the literature. But I do hope that next time round the book gets a thorough revision to bring it into the $1980 \mathrm{~s}$; it certainly deserves it.

Sam Shuster is Professor of Dermatology at the University of Newcastle upon Tyne.

\section{Superficial science}

\section{J.E. Inglesfield}

Surface Physics, 2nd Edn.

By M. Prutton.

Clarendon: 1983. Pp.138.

Hbk £12.50; $p b k £ 6.95$.

EVEN before starting to study the chemisorption of carbon monoxide on nickel, a fledgling surface scientist (unless he is a theoretician) will have to be familiar with vacuum techniques, Auger spectroscopy and the theory of low-energy electron diffraction. Professor Prutton's Surface Physics is an ideal guide for such a student in his first few terms, introducing him to the techniques used for surface analysis and structure determination, and the main concepts in surface electronic structure, thermal motion of surface atoms and adsorption. Detail is eschewed, but the account is commendably clear and there is an up-to-date list of references.

One of the remarkable features of surface science is the variety of techniques which are routinely used to unravel surface structure. This, quite justifiably, is the topic of the longest chapter in the book, and brief but lucid accounts are given of low-energy electron diffraction (LEED), reflection high-energy electron diffraction (RHEED), field ion microscopy (FIM), ion scattering, photoelectron diffraction and surface-extended X-ray absorption fine structure (SEXAFS). These techniques are based on a whole range of physics, from solving the electronic Schrodinger equation in LEED and SEXAFS to following the classical trajectories of $\mathrm{Ar}^{+}$ions with kilovolt energies in ion scattering the physics of the techniques is briefly but clearly explained. We can expect more techniques (and more confounded acronyms) in the third edition - surely on the way are grazing incidence $X$-ray diffraction, low-energy atom diffraction, and most impressive of all, the scanning tunnelling microscope.

The drawback of a book such as this is that it is inevitably bitty. This makes me less sure about its use by undergraduates. An excellent final-year undergraduate course can certainly be based on surface science, as it provides so many examples of physical principles - particularly quantum mechanics - in action. For example, the electron beam in a LEED experiment will be completely reflected by the surface when its energy coincides with a forbidden energy gap in the bulk band structure, because at such an energy electrons cannot propagate inside the solid. The concept of energy gaps leads to surface states wavefunctions localized at the surface because their energies lie in a bulk gap below the vacuum zero of energy - of which the dangling bonds on a semiconductor surface provide one example. In the space available Professor Prutton can only touch on topics such as surface states, and the unifying role of surface electronic structure has to be neglected. Undergraduates will find the book useful when they want to find out how surface states have been found experimentally.

Anyone beginning a career in a surface science lab will find this little book invaluable. Theoreticians can survive a bit longer than experimentalists without knowing what an ultra-high vacuum is, or what a LEED apparatus looks like, but they cannot get by indefinitely and they too ought to read this book when they begin research. One of the real delights of surface science is that it is still possible to understand most of what's going on, and this book provides an excellent piece of elementary education in the subject.

J.E. Inglesfield is Head of the Theory and Computational Science Division at the Science and Engineering Research Council's Daresbury Laboratory, Warrington. 